\title{
Robotic Solutions and Mechanisms for a Semi-Autonomous Endoscope
}

\author{
A. Menciassi ${ }^{1}$, Jong H. Park ${ }^{2}$, S. Lee ${ }^{2}$, S. Gorini ${ }^{1}$, P. Dario ${ }^{1}$, Jong-Oh Park ${ }^{3}$ \\ (1) Scuola Superiore Sant'Anna-CRIM \\ Piazza Martiri della Libertà 33 - 56127 PISA, Italy \\ (2) School of Mechanic al Engineering, Hanyang University \\ 17 Haengdang-Dong, Sungdong-Ku, 133-791, Seoul, Korea \\ (3) Intelligent Microsystem Center \\ Cheongryang, 130-650, Seoul, Korea

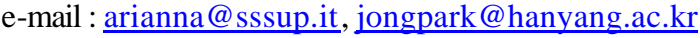

\begin{abstract}
In this paper the authors illustrate the development of a semi-autonomous robot for colonoscopy. In particular they focus on two problems: the generation of an effective and reliable advancement in the colon, and the possibility to steer the robot in order to overcome acute intestinal bends. Both problems are present also during a traditional colonoscopy, but they can be solved with an external pushing action produced by the endoscopist. The main feature of this work is the attempt to replicate the effects of external forces (generated by the medical doctors) by using just internal actions (generated by the robotic devices).

The robotic solutions and the mechanisms illustrated in this paper could be in principle integrated in an "all inside" device, with electrical wires and service tubes, but without structural cables or rigid tails which are currently used for the advancement and the orientation of traditional colonoscopes.
\end{abstract}

\section{Introduction}

Many diagnoses of important pathologies are performed currently by exploiting minimally invasive techniques which allow medical doctors to introduce endoscopes in the human body through natural orifices or small incisions. In particular, the diagnosis of colon cancer is performed by using a colonoscope which has onboard a CCD camera, bundles of optical fibers for illumination, several working channels for air, water and miniature wire-actuated instruments for local treatment and biopsy [1].

A traditional colonoscope works as a volumetric pump (Figure 1): the pushing action performed by the medical doctor is directly transformed in a real advancement of the colonoscope inside the intestine. When the medical doctor approaches an acute intestinal bend, he/she steers the endoscope tip in order to find the colon lumen and then he/she stretches the colon from outside in order to align the intestine with the colonoscope and to advance.
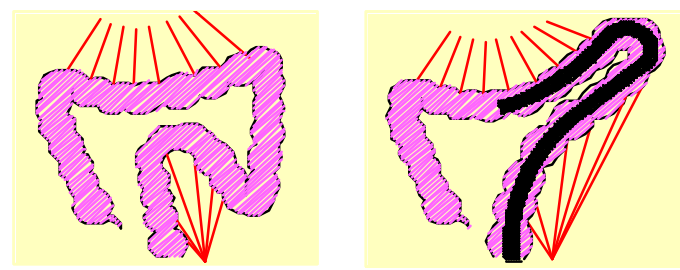

Figure 1: On the left: scheme of the human colon in normal conditions. On the right: human colon

stretched by a traditional colonoscope. The lines indicate the mesentery muscles which support elastically the colon wall

The introduction of a self-propelled device which generates just "internal" forces and which does not need any external pushing actions would improve the colonoscopy procedure in terms of patient pain reduction, easiness of advancement, "gentleness" of the entire diagnosis.

In this paper the authors first analyze the main issues related to the design of a robotic semi-autonomous device for colonoscopy (paragraph 2). Thus they present and discuss some technical implementations for the locomotion mechanism of the device (paragraph 3 and 4) and for its steering (paragraph 5). When possible, the implemented solutions are validated by in vitro and in vivo tests.

\section{Design principles for a semi-autonomous colonoscope}

In order to design a really usable robotic solution for semi-autonomous colonoscopy, we should compare advantages and drawbacks of the traditional colonoscopic procedure and the proposed colonoscopic procedure.

The advantages of traditional colonoscopy are mainly the fixed external reference during the introduction phase and the possibility to generate high forces to overcome the acute bends of the collapsed and 
tortuous intestine. On the other hand, semiautonomous colonoscopy poses several problems: no external references exist and, because of the tribological properties of the colon, slippage on the tissue occurs. Moreover, the poorly supported intestine is deformed when "internal forces" are generated in order to obtain a "self-pushed" solution; this deformation makes difficult to overcome acute bends.

The aim of a robotic semi-autonomous solution is replicating the traditional colonoscopy performance by exploiting a "gently" procedure [2 - 4].

The first objective is producing an effective advancement in the gastrointestinal tract with no or negligible losses. Two possible solutions have been studied and implemented as illustrated in the following sections: the inchworm solution and the "sliding clamper solution". Both solutions aim to overcome the locomotion problems generated by the flexibility and the tortuousness of the colon by producing an efficient stroke.

\section{Propulsion mechanism based on the "inchworm" locomotion}

An inchworm mechanism consists of two types of actuators: clamper and extensor. The simplest inchworm device consists of two clampers at its ends and one extensor at its mid section [5]. The clamper is used to adhere or clamp the device onto the substrate while the extensor generates a positive displacement (stroke). Several versions of the inchworm mechanism have been studied and implemented, by changing the actuation and the configuration of the clampers and the extensor. Currently the more promising solution consists of a pneumatic bellow - serving as the extensor - and two clamping mechanisms which suck the tissue and then grasp it by closing two opposite jaws. A picture of a typical inchworm prototype is shown in Figure 2.

Primarily, the stroke is the difference in length of the extensor in its elongated and retracted states.

On the other hand, even if each inchworm phase is efficient by itself, some stroke losses are generated by the deformation of the colon tissue during the elongation and by the elasticity of the mesentery muscles during the retraction phase.

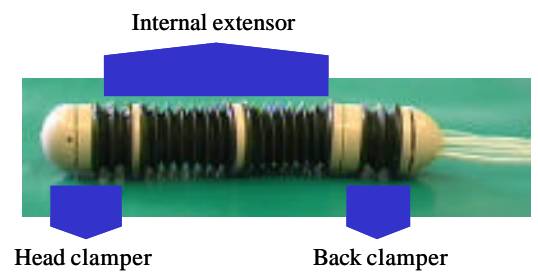

Figure 2: Typical inchworm prototype
From a first model of the colon behaviour during the inchworm locomotion [6 - 7], it results that the critical strokes which assure an effective elongation and retraction are respectively:

$$
\mathrm{S}_{\mathrm{cr}}=0,16 \times \mathrm{L}
$$

where $\mathrm{L}$ is the robot lenght, and

$$
\mathrm{S}_{\mathrm{cr}}=1,9 \times \mathrm{L}_{\mathrm{m}}
$$

where $\mathrm{L}_{\mathrm{m}}$ is the average mesentery muscles length. If we consider that mesentery muscles have an average length of $8 \mathrm{~cm}$, the average stroke necessary to advance effectively in the colon is approximately 16 $\mathrm{cm}$.

The two configurations of the inchworm device used in the model are illustrated in Figure 3.

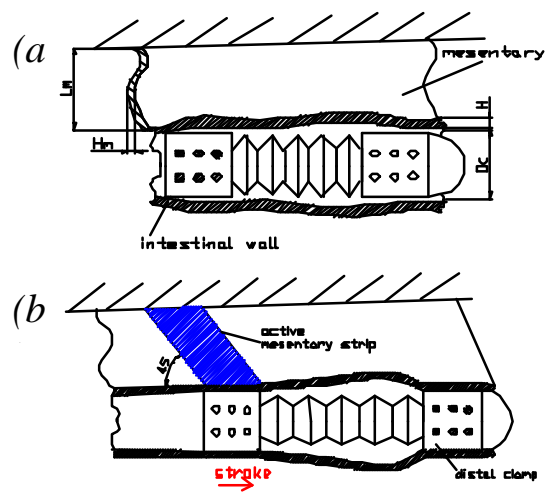

Figure 3: (a) Model of the elongation phase in the intestine with some interference between the device and the colon wall. (b) Model of the retraction phase with the mesentery resistance

\section{Propulsion mechanism based on the "sliding clampers" locomotion}

An alternative solution to obtain an efficient locomotion without fabricating "high stroke" devices consists of "generating the stroke internally", by moving the clampers. For a normal forward movement of the colonoscope, two of clampers that are located at the opposite side of the colonoscope move together as a pair. When a pair of clampers moves toward the tail of the colonoscope, the other pair moves toward the head of the colonoscope. The repetition of this motion enables the colonoscope to move forward, in the same principle that a cliff climber would move up a cliff with his/her arms.

This method of moving the clampers from the head to the tail of the device would overcome the locomotion losses caused by the flexibility and viscoelasticity of the tissue.

A CAD model and the actual colonoscope with "sliding clampers" are shown in Figure 4. 


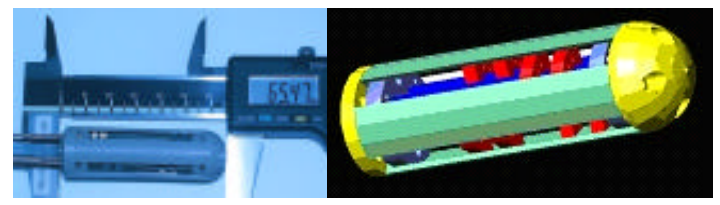

Figure 4: A CAD model and the prototype of colonoscope

The prototype of colonoscope is $65.5 \mathrm{~mm}$ long and 18 $\mathrm{mm}$ in diameter. In order to minimize the possibility of clamping failure, it has four pairs of clampers operating pneumatically. The four pairs of clampers can also give some degrees of freedom in steering the colonoscope. The body of the colonoscope is made of PEEK due to its low weight, biocompatibility, and good machinability. A tendon made of fibred wire and inserted into a long and thin stainless tube of $1.1 \mathrm{~mm}$ in diameter is used to move the clampers. The tendon is sensorised in force as illustrated in the following sub-sections.

The complete system consists of (Figure 5):

- the pneumatic subsystem with the vacuum generator, the on-off valves and a manual pressure regulator, to produce suction and insufflation;

- the motor assembly, to drive the clampers along the device body and to close them;

- 8 force sensors to measure the clamping force.

The time to complete a single motion cycle is about 10 $\mathrm{s}$; by considering the stroke length of about $28 \mathrm{~mm}$, the theoretical speed is about $17 \mathrm{~cm} / \mathrm{min}$.
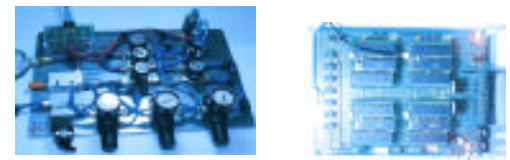

(a)

(c)

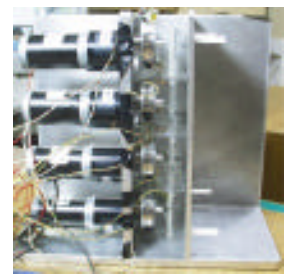

Figure 5: (a) Pneumatic circuit, (b) PIC-controller board, (c) Motor system

\subsection{Working principle of the clampers and force control}

When the colonoscope starts its operation, two pairs of clampers are forced to move toward the head of the colonoscope until a certain limit of the tendon tension is monitored. This would locate the clampers at the one end of their track. Then, the other two clampers are positioned at the other end of its track near the tail of the colonoscope. A suction process then starts to pull the tissue into the jaws of the clampers located at the head, as shown in Figure 6.
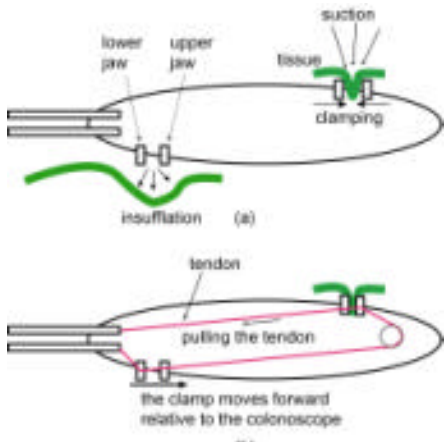

Figure 6: How the clampers of the colonoscope work

As the suction process is completed, the jaws clamp the tissue with a limited pressure, and then the clampers starts moving toward the tail of the colonoscope. The other two sets of clampers start moving toward the head of the colonoscope. This motion would force the colonoscope to move forward relative to the tissue.

Despite all the merits of clamping in terms of stable grasp and locomotion efficiency, clamping a tissue can cause serious damages to it if the clamping force is not well regulated and if some excessive clamping force is applied to it. The authors performed a few experiments with intestine tissues of pigs and estimated what the limiting clamping should be in order to avoid critical damages to the tissues.

In order to keep the clamping pressure all the time within the safe region, the authors specially designed and used force sensors with strain gauges. The method to measure the motor current directly was not adopted due to the existence of high friction at the gearbox of the actuators.

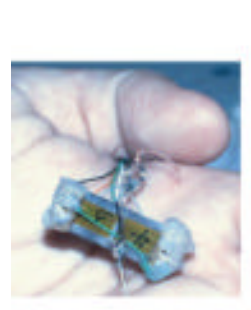

(a)

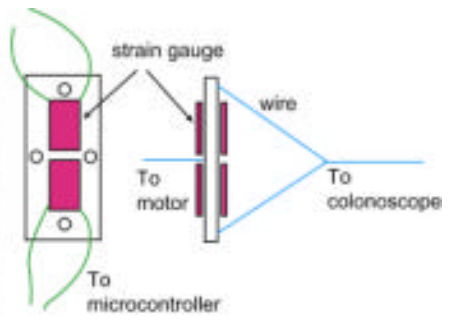

(b)
Figure 7: Force sensors integrated in the colonoscope

The force sensors used in the colonoscope are shown in Figure 7. Each sensor is a rectangular-shaped plate and is made out by duralumin. Its two ends are connected to a motor though a tendon cable and the middle part is connected to a pair of clampers through another tendon cable. The sensor measures the bending force of the sensor plate, which is then 
converted to the tension generated by the tendons.

When the jaws of a clamp move with the tissue in order to thrust the colonoscope forward, it is very critical to control the clamping force. Since the motion of each of the jaws is controlled independently, maintaining the desired level of clamping force and moving the clamp at the same time is a delicate task. If the feedback is excessively delayed, the clamping force can be so low that the tissue can be slipped out of the clamping jaws, or can be so high that the tissue can be damaged.

In order to control the motors that move tendons, to control the clamping forces and the pneumatic circuit, four 16-bit PICs were used in a hierarchical fashion. The overall block diagram of the control system is shown in Figure 8.

Force data from the force sensor were sampled at 50 $\mathrm{kHz}$ with 12-bit ADCs, resulting in a resolution of $0.01 \mathrm{~N}$. The program for PICs was written in $\mathrm{C}$ at a PC and the compiled codes were downloaded to the PICs.

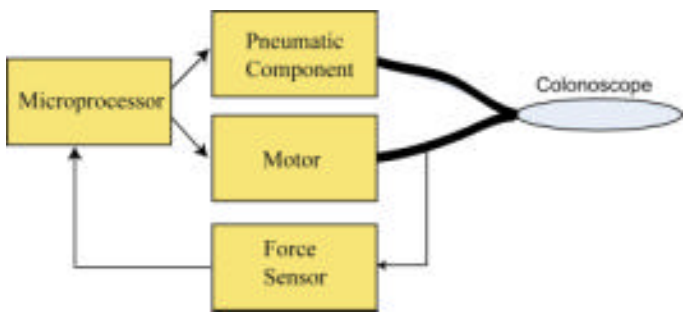

Figure 8: Overall control system for the colonoscope

\section{Steering mechanisms for colonoscopy}

During traditional colonoscopy, the medical doctors use the colonoscopic images not only to perform the diagnosis but also to assist the introduction of the device into the colon and to control its advancement. The intestine path is very tortuous and locomotion devices fail very likely when they encounter acute bends. Figure 9 shows the real curve between the descending and the transverse colon in a pig and an approximate scheme of the same curve.

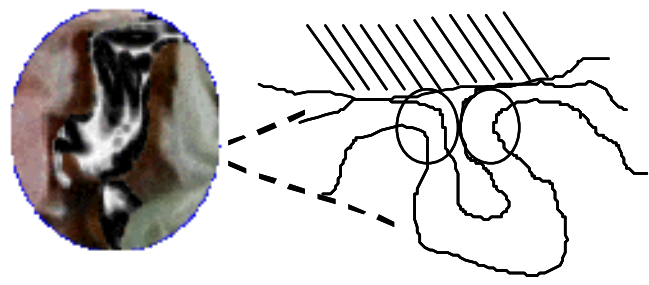

Figure 9: An example of acute bend in the pig colon and a drawing of the curve structure

On the basis of the endoscopic images, the medical doctors look for the colon lumen position and orientate the steerable tip of the colonoscope in order to follow the right direction. The tip is generally cable actuated and the medical doctors can drive it by using a knob on the colonoscope handle.

In order to replicate the traditional colonoscopic procedure, also the semi-autonomous colonoscope must be steerable [8].

Three different version of steering systems have been studied and fabricated, all with the same actuation principle (Shape Memory Alloy) but different configuration: the first one allows to bend the entire robot body; the second and the third ones are essentially separate modules to be integrated on the tip of the colonoscope in order to steer just the distal part.

\subsection{Steering the entire robot body}

This steering mechanism consists of three Shape Memory Alloy (SMA) springs inside the central bellow which can elongate and retract, thus bending the whole body of the robot. Figure 10 shows a picture of the steerable inchworm colonoscope. The reason why SMAs were selected is that they have a very high force/bulk ratio, thus allowing a light and compact design for the steering system.

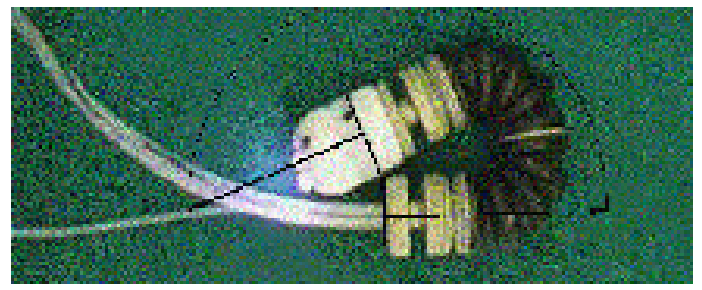

Figure 10: Steerable colonoscope integrating 3 SMA springs

Although the bending capability of the steering system is quite impressive, some important drawbacks exist. First of all, the system is slow: two air cycles (about 40 seconds) are required to cool the springs and to come back to the original position. Moreover, the SMA springs make more rigid the body even when the steering is not active and they reduce the effective stroke: $60 \mathrm{~mm}$ without the springs and $24 \mathrm{~mm}$ with the SMA springs integrated. Finally, internal air tubes must be resistant to high temperature in order to tolerate the SMA spring heating.

The system has been characterized in terms of torque: with a current supply for each SMA spring of about $1.4 \mathrm{~A}$ the maximum generated torque is $14 \mathrm{mNm}$.

Several in vitro tests on explanted pig colon have been performed in order to evaluate for which angles the steering system is absolutely necessary (Figure 11). 


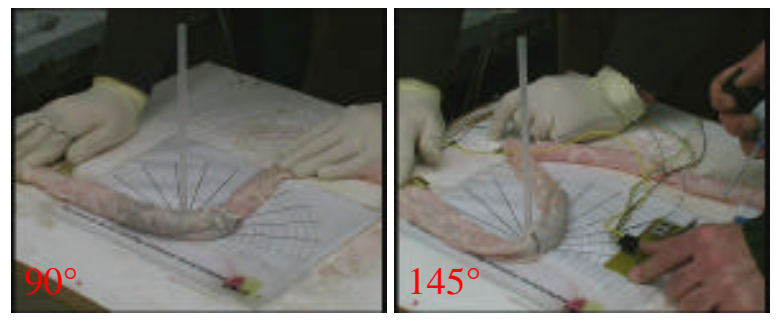

Figure 11: Some phases of the in vitro experiments carried out on the steerable inchworm device

These tests demonstrated that the system can overcome bends with angles less than $90^{\circ}$ but for angles more than $90^{\circ}$ the steering actuation is absolutely necessary to advance. The maximum angle which has been navigated in vitro was $145^{\circ}$. Thus the device was not able to proceed because of the limited stroke which was not enough to overcome the flexibility and elasticity of the tissue.

The steerable robot was tested also in vivo on a pig under anesthesia in an authorized animal laboratory with the assistance of specialized medical doctors. The device moved without actuating the steering mechanism for about $30 \mathrm{~cm}$. Then the robot stopped in an acute bend, as observed by the fluoroscopy.

The steering was activated in different attempts and the robot moved for other $10 \mathrm{~cm}$, thus demonstrating an improvement in the progression ength of about $25 \%$.

\subsection{The steerable tip}

In order to overcome the drawbacks indicated in paragraph 5.1, a steerable tip has been designed and fabricated by using a silicone bellow with length $3 \mathrm{~cm}$ containing 3 small SMA springs with a $120^{\circ}$ disposition. This configuration allows a $90^{\circ}$ bending in three directions (Figure 12) while the silicone bellow permits also a slight telescopic behavior, very useful for enhanced visualization.
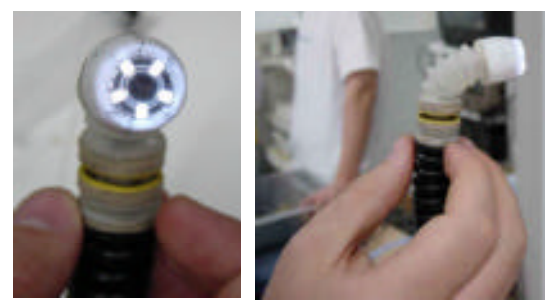

Figure 12: Steerable tip integrating a CMOS camera and leds for illumination

The SMA wire used for the springs has a diameter of $0.22 \mathrm{~mm}$ and each springs has an average diameter of $0.7 \mathrm{~mm}$ and a contracted length of $8 \mathrm{~mm}$.

In order to decrease the response time of the system a cooling system based on air re-circulation has been implemented. Cold air is introduced in the silicone bellow with a maximum pressure of 0.65 bar (tuned by a proportional valve), while the vacuum is produced through a suction hole of the same bellow. The stiffness of the tip is regulated by the differential pressure.

The maximum steering angle $\left(90^{\circ}\right)$ is achieved by a current of $0.8 \mathrm{~A}$ for each spring. The response time of the system, i.e. the time difference between the spring activation up to the maximum bending and the return to the rest position, is $3 \mathrm{~s}$. The maximum generated torque is about $9 \mathrm{mNm}$.

During three in vivo tests the robot reached $90 \mathrm{~cm}$ in the pig intestine starting from the anus and overcame two acute bends, thanks both to the steering tip and to the more flexible structure and shorter rigid part respect to the previous robots. Some fluoroscopy images taken during an in vivo test show the robot travelling an acute bend of the intestine (Figure 13).

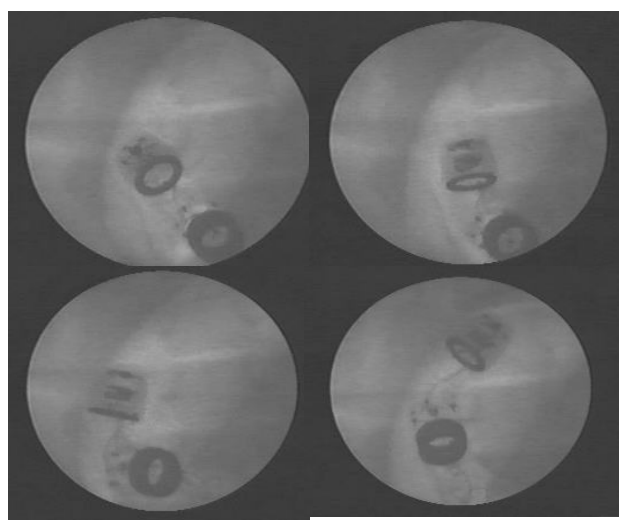

Figure 13: Sequence of locomotion during an in vivo test

\subsection{Steerable platform for the device head}

A miniature solution for the steering of the robot tip has been developed again based on SMA actuators. The prototype (Figure 15-a) consists of a distal disk which is moved by three pulling wires connected to SMA miniature springs. An unilateral spherical joint is obtained by using a ball-disk contact and three bias springs. Each SMA actuator is composed by two springs acting in parallel, in order to achieve high forces with low radial bulk. Six thin metallic rods guide internally the springs. They originally were supposed to be insulated, but after comparative measurements they have been chosen to be bare because of the relative high resistance between NiTi wire and rod. 


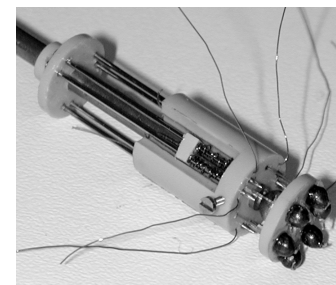

(a)

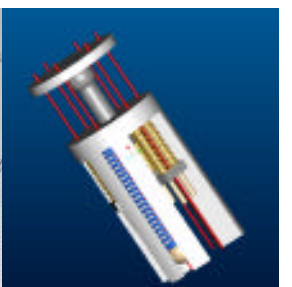

(b)
Figure 15: (a) Technical drawing of the steerable platform; (b) steerable platform prototype

The platform has got a diameter of $10 \mathrm{~mm}$ and a length of $21 \mathrm{~mm}$. The SMA springs have a length of $10 \mathrm{~mm}$ and a stiffness (when excited) of $1 \mathrm{~N} / \mathrm{mm}$.

The prototype platform is shown in Figure 15-b. Materials are PEEK for the frame and KEVLAR for the wires.

The theoretical performance for the platform were 6 $\mathrm{mm}$ for the stroke of each SMA couple of springs, a maximum bending angle of about $45^{\circ}$ and a maximum torque of $11 \mathrm{mNm}$.

After a preliminary characterization of the real prototype, we obtained the following performance

- Max angle at $0.35 \mathrm{~A}: 28^{\circ}$

- Max torque at $0.35 \mathrm{~A}-0^{\circ}: 6,8 \mathrm{mNm}$

This second concept of steerable mechanism is suitable to be integrated in a locomo tion solution with a fixed length (e.g. the sliding clampers device). Currently, the integration is in progress.

\section{Conclusions}

In this paper the authors illustrated the robotic mechanisms which they designed and fabricated in order to develop a semi-autonomous device for colonoscopy. The main investigated problem is related to the effective advancement in the intestine: because of the peculiar nature of the colon which is collapsible, slippery and very tortuous, the achievement of an effective locomotion is not trivial. Two different locomotion solutions with variable and fixed length have been presented and compared. For each solution, a steering mechanism to perform the more acute intestinal bends has been designed, fabricated and, when possible, validated. The proposed devices are all characterized by the absence of structural tails which could allow an external pushing actions. All the forces needed for the advancement of the robot are generated inside the robot thus opening the possibility of a future generation of wireless capsule-like devices for endoscopy.

\section{Acknowledgments}

The authors wish to acknowledge that this paper is a result of the research accomplished with the financial support of the Intelligent Microsystem Center, Seoul, Korea, which is carrying out one of the 21 st century's New Frontier R\&D Projects sponsored by the Korea Ministry of Science \& Technology (www.microsystem.re.kr). The authors wish to thank M.G. Trivella, MD, Prof. P. Spinelli, MD and Prof. S.Y. Song MD for their medical support and consultancy. A special thank is for Mr. Arena and Mr. Pernorio for their technical help.

\section{References}

[1] P. B. Cotton and C. B. Williams, Practical Gastrointestinal Endoscopy. Oxford: Blackwell Scientific Publications, 1990.

[2] T. Fukuda, H. Hosokai and M. Uemura, "Rubber gas actuator driven by hydrogen storage alloy for in-pipe inspection mobile robot with flexible structure," in Proc. 1989 IEEE Int. Conf. Robot. Automat., Scottsdale, AZ, May 1989, pp. 1847-1852.

[3] A. B. Slatkin, J. Burdick, W. Grundfest, "The development of a robotic endoscope," in Experimental Robotics IV, The 4th Int. Symp., Berlin Springer-Verlag, pp. 161-169, 1997.

[4] G. Iddan, G. Meron, A. Glukhovsky and P. Swain, "Wireless Capsule Endoscopy”, Nature, vol. 405, no. 6785, p. 417, 2000.

[5] P. Dario, M. C. Carrozza and A. Pietrabissa, "Development and in vitro tests of a miniature robotic system for computer assisted colonoscopy," Journal of Computer Aided Surgery, vol. 4, pp. 4-14, 1999.

[6] L. Phee, D. Accoto, A. Menciassi, C. Stefanini, M. C. Carrozza, P. Dario, "Analysis and Development of Locomotion Devices for the Gastrointestinal Tract", IEEE Transactions on Biomedical Engineering, in press.

[7] P. Dario, P. Ciarletta, A. Menciassi, B. Kim, "Modelling and experimental validation of the locomotion of endoscopic robots in the colon", ISERO2 International Symposium on Experimental Robotics, July 8-11, 2002 (submitted and under evaluation).

[8] S. J. Phee, W. S. Ng, I. M. Chen, F. Seow-Choen and B. L. Davies, "Locomotion and steering aspects in automation of colonoscopy," IEEE Engineering in Medicine and Biology Magazine, vol. 16, no. 6, pp. 85-96, 1997. 